\title{
Editorial
}

Nutrition\& Metabolism

Published online: November 21, 2018

\section{Front-of-Pack Labeling: Tool for Noncommunicable Diseases Prevention}

\author{
Visith Chavasit Wantanee Kriengsinyos \\ Institute of Nutrition, Mahidol University at Salaya, Nakhonpathom, Thailand
}

Other than the clearly unsafe food products, it is not easy for consumers to judge whether a food item is "healthy" or "unhealthy." To lower the risk of noncommunicable diseases (NCDs), consumers must be knowledgeable enough to choose foods that are appropriate for their health statuses, which is why nutrition education is globally accepted as an efficient strategy for preventing NCDs [1]. Strategies such as food-based dietary guidelines and the nutrition information table on the back of food packaging (BOP) have been implemented, which are in fact informative but for many consumers are too complicated and cannot be easily understood. Consequently, these tools cannot significantly improve eating behaviors [2]. The idea to simplify the informative nutrition table on BOPs led to the concept of front of pack labeling (FOP) that emphasizes NCDs-related nutrients, that is, energy, fat, saturated fat, sodium and sugar, as well as being more visible and easy for consumers to understand and use. Generally, 2 types of FOP are found on food products in the market, namely, nutrient-specific and summary. The nutrient-specific type indicates only certain nutrients selected from the BOP with and without guidance on health impact. The guidance is based the nutrient contents per $100 \mathrm{~g}$ or $\mathrm{mL}$ and shown simply as in a traffic light pattern.
From the guideline, food products can then be classified as low, potential or high risk for NCDs due to which nutrient based on the traffic light color [3]. Examples of the nutrient-specific FOP are monochrome and multichrome Guideline Daily Amounts (GDAs). For less knowledgeable consumers, the multichrome GDAs can be confusing, especially when they must make a judgment based on a series of different colors. Monochrome GDA has already been mandated in many countries, while the multichrome GDAs are recommended for the European Union member countries by the United Kingdom. In our opinion, both types of GDAs can be classified as a neutral approach strategy, since the provided information is not conclusive enough for consumers to make buying decisions. However, FOP can also be more directive, that is, it can be either a positive or negative approach. The "Summary-type" FOP is one example of these positive and negative approaches. The positive-approach summary-type FOP is normally used on a voluntary basis. A product with a qualified nutrient profile can show a designated symbol on the FOP. Unlike the nutrient-specific type, its qualification criteria are based on each product's nutrient profile, not just based on the single guideline [4]. In many instances, the criteria are developed by co-con-

\section{KARGER}

(c) 2018 S. Karger AG, Basel

E-Mail karger@karger.com

www.karger.com/anm
Visith Chavasit

Institute of Nutrition

Mahidol University at Salaya

Phutthamonthon, Nakhonpathom 73170 (Thailand)

E-Mail vchavasit@gmail.com 
sidering factors such as product safety, product nature, and culture. Consequently, a specific product may not be qualified to be labeled a healthy product, and the term "healthier" is more preferred. Examples of the FOP logos of this positive approach have been implemented in many countries, such as healthier choice of Singapore, healthier logo of Thailand, healthier choice of the Netherlands, key hole of Sweden, and so on [5]. In fact, the positive-approach summary-type FOP is a type of nutrient claim and the improper use of logos resulting in misleading health claims is a concern. On the contrary, the negative-approach summary-type FOP uses undesirable nutrients in a product as an educational tool for consumers. This negative approach however uses a single guideline to judge if a food product contains excessive amounts of undesirable nutrients, which must be labeled with a warning sign or message on FOP. This approach has been implemented in some Latin American countries. Without additional nutrition information, these summary type-FOPs may not be informative enough to educate consumers, which in fact is the paramount of the FOP implementation [6]. The private sector is the other key factor for the success and sustainability of an FOP program, since the more the foods of healthier nutrient profiles are available in the market, the more the visibility of the labeling. As compared to the other approaches, the positive approach has significantly brought more industrial food products with healthier nutrient profiles into the markets. However, the visibility and use of the FOPs by consumers are still the problems of many countries. After different countries in different regions use different strategies for some time, it will be interesting to evaluate their successes, especially in changing consumer behavior and increasing healthier food products on the market.

\section{Disclosure Statement}

The authors have no conflicts of interest to disclose.

\section{References}

1 WHO: Global Strategy on Diet, Physical Activity and Health, Resolution of the Fifty Seventh World Health Assembly WHA57.17, 2014.

2 Cecchini M, Warin L: Impact of food labelling systems on food choices and eating behaviours: a systematic review and meta-analysis of randomized studies. Obes Rev 2016;17: 201-210.

3 Crosetto P, Muller L, Ruffieux B: Helping consumers with a front-of-pack label: num- bers or colors? Experimental comparison between guideline daily amount and traffic light in a diet-building exercise. J Economic Psychol 2016;55:30-50.

4 Van Der Bend D, Van Dieren J, Marques MD, Wezenbeek NL, Kostareli N, Rodrigues PG, Verhagen H: A simple visual model to compare existing front-of-pack nutrient profiling schemes. Eur J Food Res Rev 2014:4:429-534.

5 Chavasit V, Kriengsinyos W, Ditmetharoj M, Phaichamanan M, Singsoong K, Sirichakwal
P, Rojjanawanicharkorn A: Nutrition labelling: educational tool for reducing risks of obesity-related non-communicable diseases; in Gordeladze JO (ed): Adiposity-Epidemiology and Treatment Modalities. Croatia, IntechOpen, 2017, pp 189-217.

6 Kees J, Royne MB, Cho YN: Regulating frontof-package nutrition information disclosures: a test of industry self-regulation vs. other popular options. J Consumer Affairs 2014;48: 147-174. 